\title{
The impact of non grey atmospheric treatment and convective modelling on the solar structure
}

\author{
Valentina Penza ${ }^{1,2}$ and Paolo Ventura ${ }^{2}$ \\ ${ }^{1}$ Universitá di Roma "Tor Vergata", via della Ricerca Scientifica 1, 00133 Roma, Italy \\ email: penza@roma2.infn.it \\ ${ }^{2}$ INAF - Osservatorio Astronomico di Roma; Via Frascati 33, 00040 MontePorzio \\ Catone - Italy \\ email: ventura@oa-roma.inaf.it
}

\section{Introduction}

The comparison between the observed and theoretical p-mode oscillation frequencies of many orders and degrees allows a detailed determination of the internal solar structure (Christensen-Dalsgaard et al. 1996). The most recent analysis shows that one source of error in the modern description of the Sun is located in the convective layers close to the surface, in the superadiabatic region (Gabriel 1994).

We investigate the role played by the convective modeling and the atmospheric treatment on the thermodynamic structure of the subphotospheric solar region. We discuss the necessity of a non grey approach when matching the internal integration with the surface, and compare the relative impact of non-greyness and the treatment of convection.

\section{The evolution code}

The evolutionary sequences presented here were calculated by means of the stellar evolution code ATON, a full description of which can be found in Ventura et al. (1998). We modeled convection with the Full Spectrum of Turbulence (FST, Canuto \& Mazzitelli 1991) model, where for the mixing length we use the expression

$$
\Lambda=z+\beta H_{p}
$$

where $z$ is the distance from the nearest convective boundary. $\beta$ is a free parameter, calibrated to reproduce the evolution of the Sun. The term $\beta H_{p}$, simulating some overshoot into the radiative region, adds a flavour of non locality to the FST treatment.

For that concerning the atmosphere model, we explore the effects of changing $\beta$. Heiter et al. (2002) and Montalban et al. (2004) implemented the FST treatment into ATLAS9 (http://ams.astro.univie.ac.at/nemo/dvd), and calculated atmosphere grids (NEMO) by using a unique $\beta$. In this work we take the outer radiative atmospheric region of NEMO models, and change the convective zone by recalculating the gradient according to the FST, but with different $\beta$ values.

\section{FST non grey models: the role of $\beta$}

We built three grids FST atmospheres with $\beta=0.09,0.15,0.18$, in the range $5000 \mathrm{~K} \leqslant$ $T_{\text {eff }} \leqslant 6000 \mathrm{~K}$ and $4.0 \leqslant \log (\mathrm{g}) \leqslant 5.0$. The $\beta$ used in the interior was chosen for each case to have a radius $R_{\odot}=6.9599 \times 10^{10} \mathrm{~cm}$ at the present age of the Sun, i.e. $4.57 \times 10^{9} \mathrm{yr}$. We also varied the optical depth of the matching point, in the range $1 \leqslant \tau_{b} \leqslant 100$. 

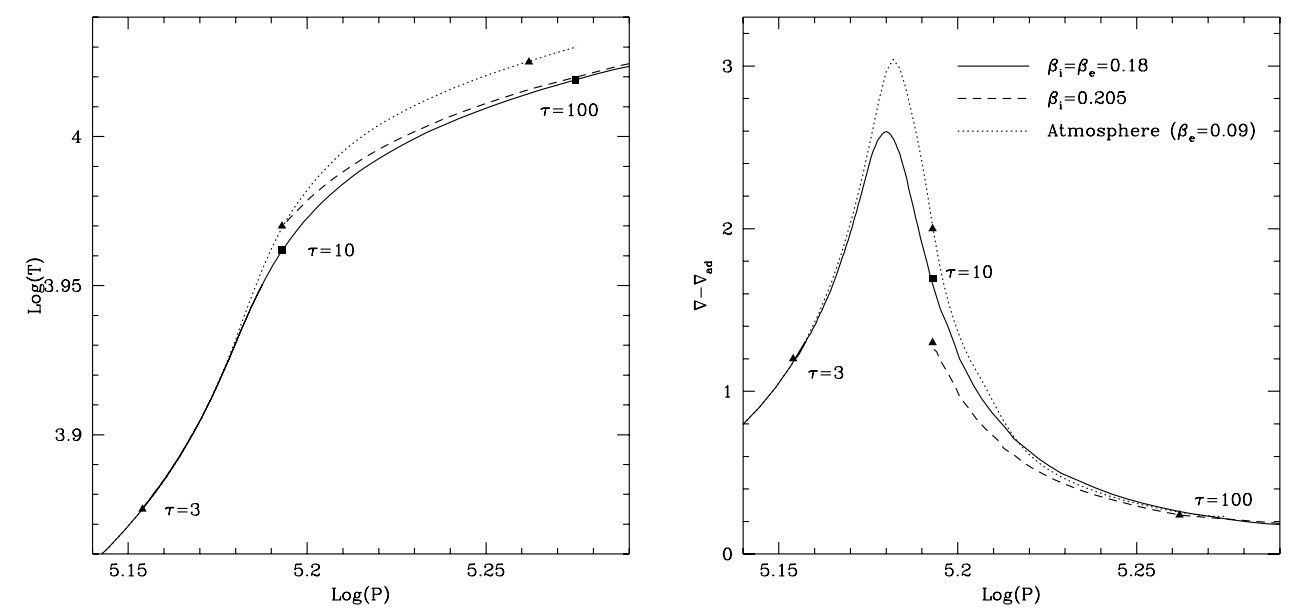

Figure 1. The subphotospheric distributions of temperature (left) and overadiabaticity (right) of two FST solar models differing for the $\beta$ chosen for the atmospheric convection modeling. Full squares and triangles mark different value for the atmospheric $\tau$, respectively, for $\beta=0.18$ and $\beta=0.09$.

In agreement with Montalban et al. (2004), we find that reproducing the evolution of the Sun is not possible when $\beta=0.09$ is used both in the interior and in the atmosphere, the effective temperature at the present epoch being too cool by $\sim 80 \mathrm{~K}$. The only way of solving the problem is changing the internal $\beta$, with a modality depending also on the chosen $\tau_{b}$. For $\tau_{b}=3,10$ we find, respectively, $\beta=0.18,0.205$; no fit can be obtained for $\tau_{b}=100$.

The two panels of Fig. 1 show the profiles of the temperature and the overadiabaticity within the subphotospheric region, for the two atmospheres $(\beta=0.09,0.18)$ and the interior. When $\tau_{b}=3$ is chosen, the results are independent of $\beta$, because the densities are so low that, though formally convective, the gradient is practically radiative in that zone. At $\tau_{b}=10$, using $\beta=0.09$ for the atmosphere leads to a larger temperature compared to $\beta=0.18$, thus requiring a higher $\beta=0.205$ in the interior to match the two profiles: in this case the solar radius is reproduced, but a large and unphysical discontinuity in the overadiabaticity is introduced at the joining point $\tau_{b}=10$ (see the right panel of Fig. 1). For $\beta=0.09$ and $\tau_{b}=100$ the fitting is not possible, because in that region the contribution of the additional term in Eq. (1) is negligible. When $\beta=0.18$ is used both in the interior and in the atmosphere, the fit turns out to be independent of $\beta$, and the gradient is smooth everywhere.

Thus, we find that when convection is modeled according to the FST scheme, it is possible to build a self-consistent solar model, in which a unique description of the convective regions is used throughout the star. We recall that this is impossible when the Mixing Length Theory (MLT, Vitense 1953) is used.

\section{The role of non-greyness}

To disentangle the role played by the atmospheric treatement, we calculated also a grey model, where the atmosphere was integrated via the Krishna Swamy (1966) law, matched with the interior at $\tau_{b}=2 / 3$. In Fig. 2 we compare the structure of the non-grey models presented in the previous section, versus the grey model, for which a value $\beta=0.16$ was 

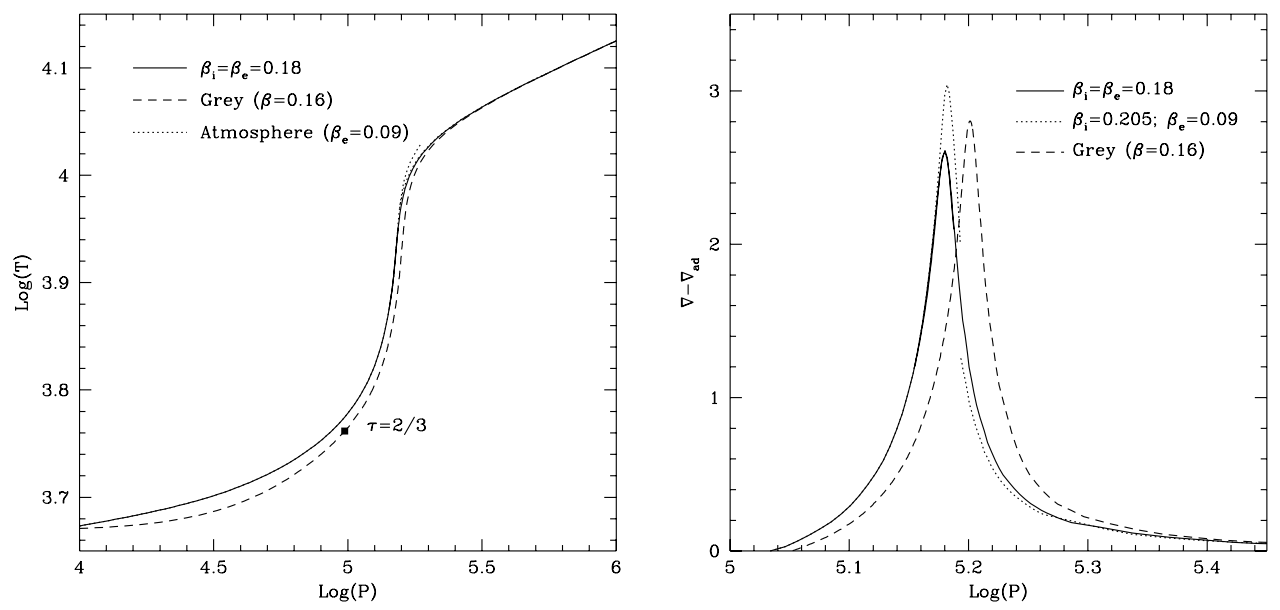

Figure 2. The same as Fig. 1, but in this case including also the grey model, calculated with $\beta=0.16$. We note that the effect of non-greyness is much more relevant of the choice of $\beta$ in determining the thermodynamic structure of the subphotospheric region.

needed to fit the solar radius. The two non-gray structures are almost indistinguishable on this scale.

We note that the point where we have the steep increase of temperature is more internal in the grey case, because in the outermost layers the slope of the P-T relation is much lower; the grey model is therefore cooler and more opaque, and consequently will have a larger pressure at $\tau=2 / 3$. In terms of overadiabaticity, we see in the right panel of Fig. 2 that, though the height of the maximum of the grey model is intermediate between the two non-grey cases, its location is found more internal, which is the reason of the differences in the thermodynamic structure distinguishing the grey from the non-grey cases, shown in the left panel of Fig. 2.

\section{Conclusions}

We present solar models where convection was treated within the FST framework, and explore the impact of the non-grey treatment of convection on the structure of the subphotospheric regions.

We find that, at odds with the MLT case, FST modeling allows the possibility of obtaining a solar model where the same convective treatment is used in the internal integration and in the atmosphere.

The role played by the parameter $\beta$ included in the FST formalism is found to be much more marginal than the non-greyness of the atmospheric treatment.

\section{References}

Canuto, V.M. \& Mazzitelli, I. 1991, ApJ 370, 295

Christensen-Dalsgaard, J., et al. 1996, Science 272, 1286

Gabriel, M. 1994, A\&A 292, 281

Heiter, U., Kupka, F., van't Veer-Menneret, C., et al. 2002, A\&SA 392, 619

Montalban, J., D’Antona, F., Kupka, F. \& Heiter, U. 2004, A\&A 416, 108

Ventura, P., Zeppieri, A., D’Antona, F. \& Mazzitelli, I. 1998, A\&A A 334, 953

Vitense, E. 1953, ZfA 32, 135 